\title{
Brain White Matter Involvement in Hereditary Spastic Paraplegias: Analysis with Multiple Diffusion Tensor Indices
}

\author{
G. Aghakhanyan, A. Martinuzzi, F. Frijia, M. Vavla, H. Hlavata, A. Baratto, N. Martino, G. Paparella, and D. Montanaro
}

\begin{abstract}
BACKGROUND AND PURPOSE: The hereditary spastic paraplegias are a group of genetically heterogeneous neurodegenerative disorders, characterized by progressive spasticity and weakness of the lower limbs. Although conventional brain MR imaging findings are normal in patients with pure hereditary spastic paraplegia, microstructural alteration in the cerebral WM can be revealed with DTI. Concomitant investigation of multiple intrinsic diffusivities may shed light on the neurobiologic substrate of the WM degeneration pattern in patients with pure hereditary spastic paraplegia across the whole brain.
\end{abstract}

MATERIALS AND METHODS: Tract-based spatial statistics analysis was performed to compare fractional anisotropy and mean, axial, and radial diffusivities of the WM skeleton in a group of 12 patients with pure hereditary spastic paraplegia and 12 healthy volunteers. Data were analyzed counting age and sex as nuisance covariates. The threshold-free cluster-enhancement option was applied, and the family-wise error rate was controlled by using permutation tests for nonparametric statistics.

RESULTS: In pure hereditary spastic paraplegia, group widespread fractional anisotropy decreases and radial diffusivity and mean diffusivity increases $(P<.05$, corrected) were found. No voxelwise difference was observed for the axial diffusivity map. Percentage of voxels within the WM skeleton that passed the significance threshold were $51 \%, 41.6 \%$, and $11.9 \%$, respectively, for radial diffusivity, fractional anisotropy, and mean diffusivity clusters. An anteroposterior pattern with preferential decrease of fractional anisotropy in the frontal circuitry was detected.

CONCLUSIONS: In patients with pure hereditary spastic paraplegia, alterations in multiple DTI indices were found. Radial diffusivity seems more sensitive to hereditary spastic paraplegia-related WM pathology and, in line with the lack of axial diffusivity changes, might indicate a widespread loss of myelin integrity. A decrease of fractional anisotropy alone in the frontal circuitry may reflect subtle disruption of the frontal connections.

ABBREVIATIONS: $A D=$ axial diffusivity; FA = fractional anisotropy; FMRIB = Functional MRI of the Brain; FSL = Functional MRI of the Brain Software Library; HSP = hereditary spastic paraplegia; $M D=$ mean diffusivity; $\mathrm{PHSP}=$ pure hereditary spastic paraplegia; $\mathrm{RD}=$ radial diffusivity; TBSS = tract-based spatial statistics

$\mathbf{T}$ he hereditary spastic paraplegias (HSPs), also called familial spastic paraparesis or Strümpell-Lorrain disease, represent a genetically and clinically heterogeneous group of neurodegenerative

Received November 7, 2013; accepted after revision January 7, 2014

From the Institute of Life Sciences (G.A.), Scuola Superiore Sant'Anna, Pisa, Italy; Neuroradiology Unit (G.A., F.F., H.H., D.M.), Fondazione CNR/Regione Toscana G. Monasterio, Pisa, Italy; Medea Scientific Institute (A.M., M.V., G.P.), Conegliano and Bosisio Parini, Treviso, Italy; and Radiology Unit (A.B., N.M.), MRI Unit, ULSS7, Conegliano, Treviso, Italy.

This work was supported by the Italian Ministry of Health (RFM 2006, RC 10.02).

Paper previously presented in part as a scientific poster at: Annual Meeting of the American Society of Neuroradiology, May 18-23, 2013; San Diego, California.

Please address correspondence to Domenico Montanaro, MD, Fondazione CNR/ Regione Toscana G. Monasterio, U.O. Neuroradiologia, Via G. Moruzzi 1, 54123 Pisa (PI), Italy; e-mail: domont@ftgm.it

- Indicates open access to non-subscribers at www.ajnr.org

三 Indicates article with supplemental on-line tables.

http://dx.doi.org/10.3174/ajnr.A3897 disorders. ${ }^{1}$ The main clinical feature is progressive spasticity due to slowly progressing "dying back" axonal degeneration, which is maximal in the terminal portions of the longest descending and ascending tracts. ${ }^{2}$ On the basis of clinical symptoms, HSPs are classified into pure or uncomplicated, in which the spastic paraplegia is the major clinical manifestation; and complex or complicated forms, presenting with additional neurologic signs, such as intellectual disability or cognitive decline, deafness, cerebellar ataxia, epilepsy, dysarthria, peripheral neuropathy, optic atrophy, and visual dysfunction. ${ }^{3}$ Autosomal dominant, autosomal recessive, or X-linked inheritance is associated with multiple genes or loci and leads to genetic heterogeneity of this disorder. The HSP loci are designated as spastic paraplegia loci and are numbered 1-56 according to their discovery. ${ }^{4}$ There is scarce evidence about the epidemiology of HSP, though its prevalence is estimated at 1.27:100,000 population in Europe. ${ }^{5}$

Findings of conventional MR imaging of the brain are usually normal in pure hereditary spastic paraplegia (pHSP). In contrast, 
nonspecific findings such as cortical atrophy and subcortical and periventricular WM alterations are present in complicated HSP. ${ }^{6}$ Distinct MR imaging findings may accompany complicated HSP; for instance, a common form of autosomal recessive HSP with SPG11 mutation (linked to the 15q13-q15 chromosome) is frequently associated with a thin corpus callosum. ${ }^{7}$ Optic nerve and cerebellar atrophy may be revealed when visual symptoms and cerebellar ataxia are present. ${ }^{8}$

DTI is an efficient technique used to characterize the in vivo microstructural organization of the WM. ${ }^{9}$ The common DTI indices are fractional anisotropy (FA) (sensitive to microstructural changes and associated with the presence of oriented structures in tissue) and mean diffusivity (MD) (characterizes mean-square displacement of molecules and the overall presence of obstacles to diffusion). ${ }^{10}$ Other indices, axial diffusivity (AD) and radial diffusivity $(\mathrm{RD})$, offer suggestive elements to differentiate axonal injury and demyelination. ${ }^{11}$ To extend our knowledge of the neurobiologic basis of WM pathology, using multiple diffusivity matrices (FA, MD, $\mathrm{RD}$, and $\mathrm{AD}$ ) is recommended. ${ }^{12}$

The present study was set up to investigate WM alterations across the whole brain in a group of patients with pHSP with SPG4, SPG5, SPG3a, and SPG10 mutations, applying tract-based spatial statistics (TBSS) analysis with multiple DTI indices.

\section{MATERIALS AND METHODS \\ Subjects}

We recruited 12 adult patients with pHSP (mean age, $49.2 \pm 8.1$ years), from the Medea Scientific Institute in Conegliano, Treviso, Italy, and 12 healthy volunteers matched by age and sex (mean age, $48.2 \pm 5.4$ years). All patients with pHSP underwent molecular genetic studies that confirmed mutations in SPG4, SPG5, $S P G 3 a$, and SPG10 loci. Careful visual inspection of each patient's MR images was performed by 3 experienced neuroradiologists (D.M., N.M., and A.B.), and structural MR imaging evidence of tumor, infection, infarction, or other focal lesions was excluded. All patients underwent a clinical examination with detailed neurologic evaluation, including measurements of spasticity, motoricity, and autonomy. Local ethics committee approval (n 63/09CE) and written informed consent from all subjects were obtained before the study.

\section{MR Imaging Acquisition and DTI Processing}

All subjects underwent MR imaging brain scans (1.5T Achieva 2.5 XR; Philips Healthcare, Best, the Netherlands) at the MR imaging laboratory of Ospedale Civile ULSS7, Conegliano, Treviso, Italy. Diffusion-weighted images were acquired by using EPI sequences (TR/TE/TI, 10,000/69/2400 ms; bandwidth, $2117.4 \mathrm{kHz}$; acquisition matrix, $104 \times 102$; voxel size, $2 \times 2 \times 2.03 \mathrm{~mm}$; b-value $=0$ and $1.000 \mathrm{~s} / \mathrm{mm} ; 102$ contiguous sections; 32 different gradient directions).

DTI processing was performed by using FSL, Version 4.1.1 (http://fsl.fmrib.ox.ac.uk/fsl). ${ }^{13}$ First, raw diffusion data were corrected for motion artifacts and eddy current distortions; then, brain was extracted by using the Brain Extraction tool. The FMRIB Diffusion Toolbox, Version 2.0 (http://www.fmrib.ox.ac.uk/ $\mathrm{fsl} / \mathrm{fdt} /$ index.html), was used to fit the diffusion tensor and compute the diagonal elements $(\lambda 1, \lambda 2$, and $\lambda 3)$ at each brain voxel, from which the derived metrics $\mathrm{AD}, \mathrm{MD}$, and FA were also inferred. We calculated the RD matrix for each subject, averaging $\lambda 2$ and $\lambda 3$ diagonal elements of the diffusion tensor [RD $=1 / 2 \times$ $(\lambda 2+\lambda 3)]$.

\section{Tract-Based Spatial Statistics Analysis}

Voxelwise statistical analysis of the FA, MD, $\mathrm{RD}$, and $\mathrm{AD}$ data were performed by using TBSS. ${ }^{14}$ All subjects' FA data were aligned into a common space by using the Nonlinear Registration Tool in FMRIB. The FMRIB58-FA standard-space image was used as a target image following the recommendations of the FSL software guidelines. Next, the mean FA image was created and thinned (threshold FA value of 0.25 ) to create a mean FA skeleton, which represented the centers of all tracts common to the group. Finally, all subjects' spatially normalized FA, AD, RD, and MD data were projected onto the skeleton, and resulting data were fed into voxelwise cross-subject statistics.

\section{Conjunction Map}

To obtain a spatial distribution pattern of FA and RD changes, we created a conjunction map. First, resulting FA and RD maps of the voxelwise statistics were thresholded at $P=.05$, corrected for multiple comparisons and then binarized and masked. The fslmaths program (part of FSL, http://www.mit.edu/ satra/nipype-nightly/ interfaces/generated/nipype.interfaces.fsl.maths.html), which allows mathematic manipulation of images, was used to subtract binarized and masked maps of significance for FA and RD matrices.

\section{Statistical Analysis}

Demographic and descriptive differences for continuous variables (eg, age) between HSP and control groups were examined with the Wilcoxon rank sum test; for categoric variables (eg, sex), group differences were evaluated by using the Pearson $\chi^{2}$ test. Statistical analyses were performed by using R software, Version 2.15.1 (http://www.r-project.org/).

DTI-based voxelwise statistics were performed by using the program Randomize, part of FSL. Voxelwise differences among groups were assessed, applying 2-sample $t$ tests. We used the thresholded mean FA skeleton (mean value of 0.25 ), setting the number of permutations to 5000 with the threshold-free clusterenhancement option and the significance threshold at $P=.05$, corrected for multiple comparisons to control the family-wise error rate. Data were analyzed counting age and sex as a nuisance covariates.

\section{RESULTS}

Demographic and clinical characterizations are summarized in Table 1. The patients with pHSP and healthy controls did not differ in terms of age and sex distribution. All patients enlisted in our study showed a phenotype consistent with pHSP.

\section{Analysis of Multiple DTI Indices}

The mean diffusion metrics (FA, MD, AD, and RD) in the WM skeleton were extracted for each subject. Statistical analysis revealed significant group differences in all mean diffusivity indices (On-line Table 1). Compared with controls, patients with pHSP had significantly decreased mean FA $(P<.001)$, increased mean 
$\mathrm{RD}(P<.001)$, increased mean $\mathrm{MD}(P<.005)$, and a less notable increase in mean $\operatorname{AD}(P=.01)$.

Voxelwise TBSS analyses revealed widespread alterations in multiple DTI indices (Fig 1). Compared with the healthy group, patients with pHSP showed decreases of FA $(P<.05$, corrected $)$ in multiple WM regions (Fig 1A), including the bilateral anterior thalamic radiations, corticospinal tracts, corpus callosum with forceps major and minor, and parts of the inferior and superior longitudinal fasciculi. Significant clusters for each matrix were separately masked and labeled with reference to the JHU ICBMDTI-81 White Matter Labels, part of the FSL atlas tools (Table 2).

Table 1: Demographic and clinical characteristics for patients with HSP and the control group ${ }^{a}$

\begin{tabular}{|c|c|c|c|}
\hline & $\begin{array}{l}\text { Patients with } \\
\text { HSP ( } n=12)\end{array}$ & $\begin{array}{l}\text { Healthy Controls } \\
\qquad(n=12)\end{array}$ & $P$ Value \\
\hline Age at MRI (yr) & $50(49 \pm 8)$ & $48(48 \pm 5)$ & $.5^{\mathrm{b}}$ \\
\hline Sex (female) & $50 \%$ & $67 \%$ & $.4^{c}$ \\
\hline Disease duration (yr) & $23(24 \pm 16)$ & - & - \\
\hline Disease onset (yr) & $23(25 \pm 18)$ & - & - \\
\hline \multicolumn{4}{|l|}{ Spastic gate loci } \\
\hline SPG10 & $8 \%(1)$ & & \\
\hline SPG3a & $8 \%(1)$ & & \\
\hline SPG4 & $58 \%(7)$ & & \\
\hline SPG7+SPG4 & $8 \%(1)$ & & \\
\hline SPG5 & $17 \%(2)$ & & \\
\hline
\end{tabular}

a Values are median, mean \pm SD, or No. (\%). Numbers after percentages are frequencies. b Wilcoxon test.

c Pearson test.
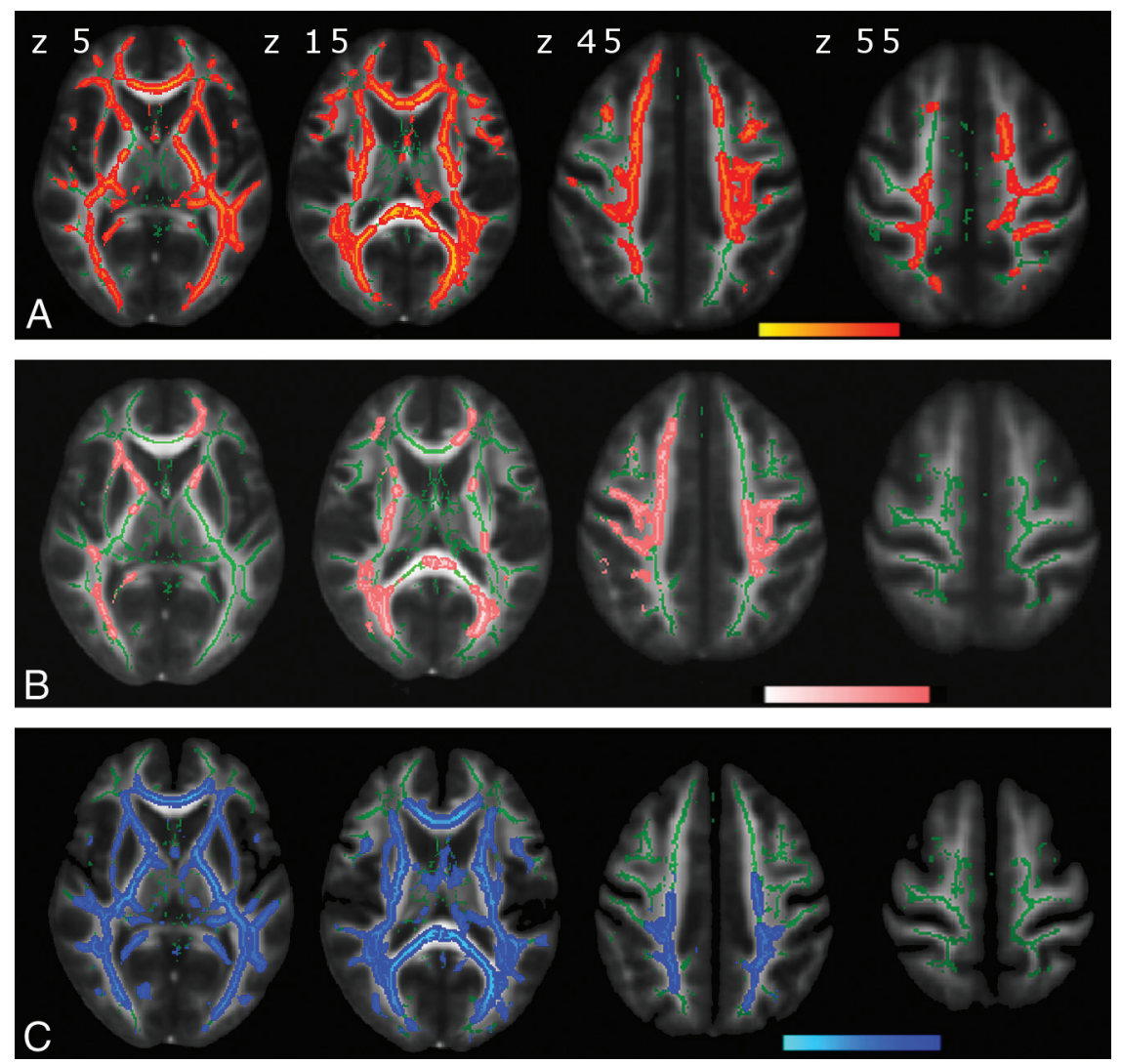

FIG 1. DTI indices maps $(P<.05$, corrected) are represented in the FMRIB58_FA template on the axial plan with z-axis coordinates (in millimeters) above the upper row images. Green corresponds to the mean white matter skeleton of all participants. A, Red-yellow represents decreased FA. B, Pinklight pink represents increased MD. C, Blue-light blue represents increased RD.
Most regions showing a decrease in FA showed a corresponding increase in $\mathrm{RD}(P<.05$, corrected, Fig $1 C)$. Changes in $\mathrm{MD}(P<$ .05 , corrected, Fig $1 B$ ) were more restricted in comparison with regions of decreased FA and increased RD. No significant differences emerged after voxelwise TBSS analysis of AD.

Percentage changes of the voxels in the WM skeleton that passed the significance threshold $(P<.05$, corrected) were $51 \%$, $41.6 \%$, and $11.9 \%$, respectively, for RD, FA, and MD clusters. All clusters consisted of a large number of voxels that did not split into smaller clusters even after increasing the significance threshold to $P<.01$. All cluster characteristics are detailed in the supplementary material (On-line Table 2).

The magnitude and topographic pattern of significant changes in multiple DTI indices were not equivalent. To address this issue, we created a conjunction map of FA and RD changes. Anterior frontal structures showed a reduction in FA, without significant changes in $\mathrm{RD}$, while posterior supratentorial brain regions and the brain stem showed a pre-eminent increase in $\mathrm{RD}$ compared with FA changes (Fig 2)

\section{DISCUSSION}

We aimed to investigate cerebral WM changes in patients with HSP, applying voxelwise analysis of multiple diffusivity indices. Accounting for the huge genetic and phenotypic variability in HSPs,${ }^{15}$ we carefully selected patients with $\mathrm{pHSP}$ with confirmed $S P G$ mutations and without structural abnormalities on MR imaging. Exploring otherwise normal-appearing cerebral WM in patients with pHSP, we found remarkable and diffuse abnormalities in WM microstructure, including a decrease in FA, an increase in $\mathrm{MD}$ and $\mathrm{RD}$, but no changes in $\mathrm{AD}$.

Currently, DTI is one of the most successful techniques among clinicians and researchers to study WM architecture. The FA index is frequently used to describe the shape of diffusion with a scalar value, while MD is used to describe the local magnitude of diffusion, regardless of direction. ${ }^{9,16}$ Overall, breakdown of WM integrity leads to a decrease in FA and/or an increase in MD. Although the FA is quite sensitive to a broad spectrum of pathologic conditions, it has been argued that a single scalar value cannot describe the full shape, orientation, and anisotropy of the WM. ${ }^{12}$ For instance, different combinations of diffusion tensor eigenvalues (which are the diagonal elements of the tensor and proportional to the squares of the lengths of the ellipsoid axes) can generate the same FA values. ${ }^{10}$ Not surprising, the apparent diffusivities in the directions parallel and perpendicular to the WM tracts, designated as the axial and radial diffusivities, become more 
appealing. ${ }^{17}$ Animal studies indicated that these measures may be selectively sensitive to specific neural changes, with $\mathrm{AD}$ reflecting axonal differences (eg, axonal damage or loss) and RD reflecting differences in the degree of myelin integrity, myelination, and demyelination. ${ }^{11,18}$

The major neuropathologic feature in HSPs is axonal degeneration, which is maximal in the terminal portions of the longest descending and ascending tracts due to the disruption of the axonal transport. ${ }^{19}$ The most severely affected pathways are the corticospinal tracts and the fasciculus gracilis. Spinocerebellar tracts may also be involved in approximately $50 \%$ of cases. ${ }^{20}$

In HSPs, the extension of the neurodegenerative process to the cerebral WM is still less known. Only a few small cohort DTI studies have demonstrated widespread alteration of multiple indices in patients with complicated HSP with a thin corpus callosum and SPG11 mutation. ${ }^{21,22}$ This form of complicated HSP is characterized by rapidly progressive spasticity of early onset, mental deterioration, and other neurologic manifestations such as seizures and cerebellar ataxia. ${ }^{23}$ Furthermore, it was shown that complicated HSPs display a different pattern of topography and severity of cerebral WM volume changes in respect to $\mathrm{pHSP}^{24}$ This finding may suggest that pure and complicated HSPs are not uniform entities and need to be explored separately.

Table 2: Suprathreshold ( $P<.05$, corrected) cluster distribution (\%) respectively for FA, MD, and RD indices

\begin{tabular}{lccc}
\hline \multicolumn{1}{c}{\begin{tabular}{c} 
JHU White-Matter Tractography \\
\multicolumn{1}{c}{ Atlas Labels }
\end{tabular}} & FA & MD & RD \\
\hline L anterior thalamic radiation & 1.27 & 1.17 & 1.54 \\
R anterior thalamic radiation & 0.81 & 0.94 & 1.01 \\
L corticospinal tract & 0.96 & 0.95 & 0.96 \\
R corticospinal tract & 1.03 & 1.40 & 1.02 \\
L cingulum (cingulate gyrus) & 0.44 & 0.28 & 0.43 \\
Corpus callosum with forceps major & 1.26 & 1.94 & 1.17 \\
Corpus callosum with forceps minor & 3.26 & 2.63 & 2.04 \\
L inferior fronto-occipital fasciculus & 1.95 & 0.40 & 1.80 \\
R inferior fronto-occipital fasciculus & 1.47 & 1.85 & 1.79 \\
L inferior longitudinal fasciculus & 0.93 & 0.30 & 1.58 \\
R inferior longitudinal fasciculus & 2.02 & 0.88 & 1.22 \\
L superior longitudinal fasciculus & 1.10 & 3.13 & 1.98 \\
R superior longitudinal fasciculus & 0.76 & 3.56 & 1.67 \\
L superior longitudinal fasciculus & 0.96 & 1.01 & 0.96 \\
$\quad$ (temporal part) & & & \\
R superior longitudinal fasciculus & 0.44 & 1.07 & 0.66 \\
$\quad$ (temporal part) & & & \\
\hline
\end{tabular}

Note:-L indicates left; R, right.

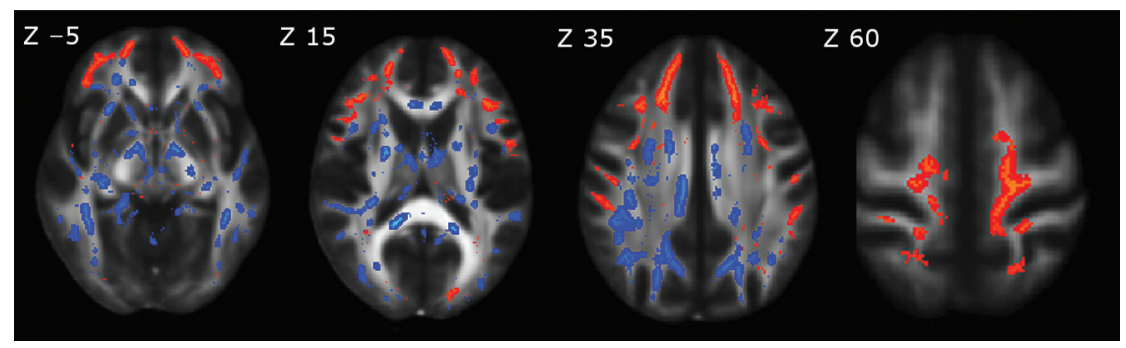

FIG 2. Conjunction map of spatial differences between reduced FA and increased RD maps, represented in the FMRIB58_FA template on the axial plane with Montreal Neurological Institute 152 coordinates (in millimeters) above each upper row image. Red-yellow represents decreased FA; blue-light blue, increased RD. A decrease in FA alone shows an anterior frontal circuitry pattern, while an increase in RD is more prominent in the posterior supratentorial brain regions and brain stem.
Meanwhile, the evidence from multiple DTI studies exploring WM alterations in different motor neuron disorders, such as amyotrophic and primary lateral sclerosis, consistently indicated a widespread decrease in $\mathrm{FA}$ and a variable increase in $\mathrm{MD}, \mathrm{AD}$, and $\mathrm{RD}$ values along motor and extramotor areas. ${ }^{25-28}$ One recent study directly linked a WM alteration pattern in patients with HSP and a group of patients with amyotrophic and primary lateral sclerosis, demonstrating a similar pattern of FA reduction in the corticospinal tracts and corpus callosum. ${ }^{29}$ Some phenotype variations of HSPs may mimic upper motor neuron disorders, but different neuropathologic elements characterize these 2 nosologic entities: axonopathy, thought to be the primary cause of neurodegeneration in HSPs, while cell bodies are relatively unaffected until late in the disease course ${ }^{1}$; and degeneration of motor neurons, on the contrary, the pathophysiologic hallmark in motor neuron disorders. ${ }^{30}$

In HSPs, axonopathy is mainly characterized by focal swelling of axons with marked accumulation of organelles and intermediate filaments associated with impaired retrograde transport. ${ }^{31}$ In addition, morphologic changes (axonal attenuation loss and decreased caliber) were observed at all levels from the lumbar to the medullar portion of the axons. ${ }^{19}$ Even though axonal damage is mainly associated with changes in diffusion properties parallel to the neuroaxis, in our patients with pHSP, we did not find changes in the $\mathrm{AD}$ map by using voxel-by-voxel analysis. To further address this issue, we extracted all mean diffusivities from the WM skeleton for each subject (On-line Table 1), and in contrast to other diffusivity indices (FA, RD, and $\mathrm{MD}$ ), we observed only a slight increase in mean $\mathrm{AD}(P=.01)$.

One possible explanation for the lack of $\mathrm{AD}$ changes may be related to complexity and temporal dynamism that occurs with axonal breakdown and clearance: alteration of axonal transport and accumulation of organelles create barriers to the longitudinal displacement of water molecules causing a decrease in AD. ${ }^{32}$ Subsequently, the cellular debris are cleared by microglia, resulting in re-establishment or even an increase in the diffusion in the longitudinal direction leading to an increase in $\mathrm{AD} .^{33}$

Myelin loss in patients with HSP is presumed to be secondary, due to axonal damage. ${ }^{19}$ As a consequence of degraded axonal membranes and disruption of myelin sheaths, the hindrance of water moving across fibers is reduced, which leads to an increase in $\mathrm{RD}^{32}$ In agreement with this feature, we found a widespread increase in $\mathrm{RD}$ in all major WM tracts, suggesting the presence of extensive demyelination in the cerebral level of patients with pHSP. ${ }^{34}$ It is difficult to conclude whether myelin loss is primary or secondary due to axonal damage. Neuropathology in HSPs is complex and combines elements of axonal loss, gliosis, and demyelination, which will result in competing influences on the diffusion tensor.

The next interesting finding emerging from our study is the inconsistency in spatial overlapping of FA and $\mathrm{RD}$ changes. Although both DTI indices were diffusively altered, in the conjunction 
map of the decreased FA and increased RD, an anteroposterior pattern was detected (Fig 2): The anterior WM seemed more susceptible to FA changes; meanwhile, the posterior supratentorial brain regions and brain stem were more prone to $\mathrm{RD}$ changes. Because the FA metric is a function of all 3 eigenvalues of the diffusion tensor, concurrent changes in parallel and perpendicular diffusivities may reduce the sensitivity of FA in the posterior brain regions. Mild microstructural alterations of the minor fibers and subtle disruption of the frontal lobe connections solely may lead to a decrease of FA in the anterior frontal circuitry. ${ }^{35}$ However, the neurobiologic substrate and neuropsychological correlates of these topographic discordances need to be further elucidated.

The study is not lacking limitations. First, we involved a limited number of patients with pHSP due to low prevalence of the disease and the strict inclusion criteria of the study. Second, our cohort is heterogeneous in terms of SPG genes and loci, but phenotype homogeneity is consistent for the group sample because we enrolled only patients with pHSP with confirmed SPG mutations. Next, TBSS analysis has many advantages, but it still has limitations in analyzing small fiber tracts and regions of crossing fibers or tract junctions. Nevertheless, TBSS is a revolutionary method for detecting group voxelwise changes in the whole brain. It is successfully designed to overcome problems with registration algorithms and arbitrariness of spatial smoothing presented in other voxel-by-voxel approaches, such as voxel-based analysis, which may crucially affect the results due to the highly directional and topographic nature of DTI. ${ }^{36}$ Furthermore, the current crosssectional design of our study cannot address longitudinal changes in correlation with disease onset, severity, and progression rate. Future longitudinal DTI studies are needed to unveil those questions.

\section{CONCLUSIONS}

We obtained a comprehensive landscape of multiple DTI matrices in patients with pHSP and demonstrated significant reductions in FA and increases in RD and MD, but no changes in $\mathrm{AD}$ in the areas of otherwise normal-appearing cerebral WM. This finding indicates that HSPs diffusely affect cerebral WM microstructure. A widespread increase in $\mathrm{RD}$ with the absence of $\mathrm{AD}$ changes may indicate extensive demyelination, whereas a decrease in FA alone in the frontal circuitry might reflect subtle disruption of the frontal lobe connections. Whether lack of AD changes reflects the temporal dynamism of the underlying neuropathologic process during which various neuronal components may be affected by different stages of the disease still needs to be elucidated.

\section{ACKNOWLEDGMENTS}

We thank all patients participating in this study and their families.

Disclosures: Andrea Martinuzzi-RELATED: Grant: Ministry of Health, Italy, ${ }^{*}$ Comments: Support for the work carried out to produce the data here reported is also provided by “Ricerca Finalizzata 2012," Support for Travel to Meetings for the Study or Other Purposes: Ministry of Health, Italy, ${ }^{\star}$ Comments: Travel funds are included in the above-mentioned grant. Dominico Montanaro-UNRELATED: Grants/ Grants Pending: Italian Ministry of Health (RF-2010-2309954). * *Money paid to the institution.

\section{REFERENCES}

1. Schwarz GA. Hereditary (familial) spastic paraplegia. AMA Arch Neurol Psychiatry 1952;68:655-62

2. Behan WM, Maia M. Strümpell's familial spastic paraplegia: genetics and neuropathology. J Neurol Neurosurg Psychiatry 1974;37:8-20

3. Harding AE. Classification of the hereditary ataxias and paraplegias. Lancet 1983;1:1151-55

4. Dürr A. Genetic testing for the spastic paraplegias: drowning by numbers. Neurology 2008;71:236-38

5. McMonagle P, Webb S, Hutchinson M. The prevalence of "pure" autosomal dominant hereditary spastic paraparesis in the island of Ireland. J Neurol Neurosurg Psychiatry 2002;72:43-46

6. Casali C, Valente EM, Bertini E, et al. Clinical and genetic studies in hereditary spastic paraplegia with thin corpus callosum. Neurology 2004;62:262-68

7. Paisan-Ruiz C, Dogu O, Yilmaz A, et al. SPG11 mutations are common in familial cases of complicated hereditary spastic paraplegia. Neurology 2008;70:1384-89

8. Depienne C, Stevanin G, Brice A, et al. Hereditary spastic paraplegias: an update. Curr Opin Neurol 2007;20:674-80

9. Basser PJ, Pierpaoli C. Microstructural and physiological features of tissues elucidated by quantitative-diffusion-tensor MRI. J Magn Reson B 1996;111:209-19

10. Le Bihan D, Mangin JF, Poupon C, et al. Diffusion tensor imaging: concepts and applications. J Magn Reson Imaging 2001;13:534-46

11. Song SK, Sun SW, Ramsbottom MJ, et al. Dysmyelination revealed through MRI as increased radial (but unchanged axial) diffusion of water. Neuroimage 2002;17:1429-36

12. Alexander AL, Lee JE, Lazar M, et al. Diffusion tensor imaging of the brain. Neurotherapeutics 2007;4:316-29

13. Smith SM, Jenkinson M, Woolrich MW, et al. Advances in functional and structural MR image analysis and implementation as FSL. Neuroimage 2004;23(suppl 1):S208-19

14. Smith SM, Jenkinson M, Johansen-Berg H, et al. Tract-based spatial statistics: voxelwise analysis of multi-subject diffusion data. Neuroimage 2006;31:1487-505

15. Fink JK. Hereditary spastic paraplegia. Neurol Clin 2002;20:711-26

16. Pierpaoli C, Barnett A, Pajevic S, et al. Water diffusion changes in Wallerian degeneration and their dependence on white matter architecture. Neuroimage 2001;13:1174-85

17. Wheeler-Kingshott CA, Cercignani M. About "axial" and "radial" diffusivities. Magn Reson Med 2009;61:1255-60

18. Xie M, Wang Q, Wu TH, et al. Delayed axonal degeneration in slow Wallerian degeneration mutant mice detected using diffusion tensor imaging. Neuroscience 2011;197:339-47

19. Deluca GC, Ebers GC, Esiri MM. The extent of axonal loss in the long tracts in hereditary spastic paraplegia. Neuropathol Appl Neurobiol 2004;30:576-84

20. McDermott C, White K, Bushby K, et al. Hereditary spastic paraparesis: a review of new developments. J Neurol Neurosurg Psychiatry 2000;69:150-60

21. França MC, Yasuda CL, Pereira FRS, et al. White and grey matter abnormalities in patients with SPG11 mutations. J Neurol Neurosurg Psychiatry 2012;83:828-33

22. Garaci F, Toschi N, Lanzafame S, et al. Diffusion tensor imaging in SPG11- and SPG4-linked hereditary spastic paraplegia. Int J Neurosci 2014;124:261-70

23. Lossos A, Stevanin G, Meiner V, et al. Hereditary spastic paraplegia with thin corpus callosum: reduction of the SPG1 1 interval and evidence for further genetic heterogeneity. Arch Neurol 2006;63:756-60

24. Kassubek J, Juengling FD, Baumgartner A, et al. Different regional brain volume loss in pure and complicated hereditary spastic paraparesis: a voxel-based morphometric study. Amyotroph Lateral Scler 2007;8:328-36

25. Kassubek J, Ludolph AC, Müller HP. Neuroimaging of motor neuron diseases. Ther Adv Neurol Disord 2012;5:119-27

26. Agosta F, Galantucci S, Riva N, et al. Intrahemispheric and inter- 
hemispheric structural network abnormalities in PLS and ALS. Hum Brain Mapp 2014;35:1710-22

27. Lombardo F, Frijia F, Bongioanni P, et al. Diffusion tensor MRI and MR spectroscopy in long lasting upper motor neuron involvement in amyotrophic lateral sclerosis. Arch Ital Biol 2009;147:69-82

28. Prudlo J, Bißbort C, Glass A, et al. White matter pathology in ALS and lower motor neuron ALS variants: a diffusion tensor imaging study using tract-based spatial statistics. J Neurol 2012; 259:1848-59

29. Müller HP, Unrath A, Huppertz HJ, et al. Neuroanatomical patterns of cerebral white matter involvement in different motor neuron diseases as studied by diffusion tensor imaging analysis. Amyotroph Lateral Scler 2012;13:254-64

30. Redler RL, Dokholyan NV. The complex molecular biology of amyotrophic lateral sclerosis (ALS). Prog Mol Biol Transl Sci 2012; 107:215-62

31. Tarrade A, Fassier C, Courageot S, et al. A mutation of spastin is responsible for swellings and impairment of transport in a region of axon characterized by changes in microtubule composition. Hum Mol Genet 2006;15:3544-58

32. Sun SW, Liang HF, Cross AH, et al. Evolving Wallerian degeneration after transient retinal ischemia in mice characterized by diffusion tensor imaging. Neuroimage 2008;40:1-10

33. Concha L, Gross DW, Wheatley BM, et al. Diffusion tensor imaging of time-dependent axonal and myelin degradation after corpus callosotomy in epilepsy patients. Neuroimage 2006;32:1090-99

34. Alexander AL, Hurley SA, Samsonov AA, et al. Characterization of cerebral white matter properties using quantitative magnetic resonance imaging stains. Brain Connect 2011;1:423-46

35. Burzynska AZ, Preuschhof C, Bäckman L, et al. Age-related differences in white matter microstructure: region-specific patterns of diffusivity. Neuroimage 2010;49:2104-12

36. Soares JM, Marques P, Alves V, et al. A hitchhiker's guide to diffusion tensor imaging. Front Neurosci 2013;7:31 